\title{
Modified Usability Test Scenario: User Story Approach to Evaluate Data Visualization Dashboard
}

\author{
Nurul Tiara Kadir ${ }^{1}$, Rudy Hartanto ${ }^{2}$, Selo Sulistyo ${ }^{3}$
}

\begin{abstract}
The data processing results are commonly displayed in a dashboard with various graphic visualization forms to deliver new knowledge easier to understand by users. However, many data analysis dashboards cannot communicate the knowledge effectively and efficiently given the unsuitable design implementation. Therefore, research to measure the interface display's effectiveness in the data analysis system is deemed necessary. This research proposed a scenario modification in the usability test with a user story approach to measuring the system interface display in delivering the information to users. The approach of a usability test with the user story is expected to be capable of helping the researcher in understanding the user habits indirectly. There were 20 participants to validate the proposed method. Participants were asked to use the system and answer several questions to develop their user experience. After developing user experience for each user, the System Usability Scale (SUS) was conducted. SUS score results obtained from this research was 75.25. Besides, the researcher also measured the understanding level among the users using questionnaires. The questionnaire results were converted into numbers and resulted in a mean value of 91.8. Those two values indicate the users' ability to use the system well and obtain the new knowledge displayed in the data analysis dashboard.
\end{abstract}

Keywords-Usability Test, Dashboard Interface, Data Visualization, User Story.

\section{INTRODUCTION}

Data analysis today has been widely developed and used for specific purposes such as social network analysis, government policies, health, and education. In the era of big data, data in a large volume have high acceleration and variation. There is a need to do an analysis at best and have a data submission skill to have meaningful knowledge from the data in the large volume [1].

The data analysis process involving the data set in a large volume to provide meaningful information is commonly used to decide. One of the important measures to achieve that is how the interface display designer plays a role in submitting the data through the visual display that is easier for the users to understand [1].

Interactive visualization in informing a finding of data processing can support the users in making a hypothesis and verification for the finding resulted from the data analysis system. Any different visualization perspectives will also help

1,2,3 Department of Electrical and Information Engineering, Faculty of Engineering, Universitas Gadjah Mada, Jln. Grafika No. 2, Kampus UGM, Yogyakarta, 55281, INDONESIA (Tel. +62-274552305; e-mail: 'Inurul.tiara.k@mail.ugm.ac.id, ${ }^{2}$ rudy@ugm.ac.id, ${ }^{3}$ selo@ugm.ac.id. broaden the understanding of the users about the different data results

Commonly, the application of the data analysis is made to help in the decision making. Even though the data analysis results can result in the high accuracy of the information, the data submission which is not well designed will lead to communication failure between the system and the users. As the consequence, it makes the received information uncompleted and leads to failure in the understanding of knowledge.

In designing a product, it needs an understanding of the prospective users purposely to result in a product suitable for the users' needs. One of the ways to reach that matter is through the process of User Experience [2]. Providing a good User Experience becomes one of the important components in designing the data analysis system. It can provide the results of the data analysis accurately and must be successful in communicating the information resulted informatively to the users.

In some research, data visualization in the interface display of data analysis system is a domain that is not discussed in detail. The measurement in the interface display's effectiveness, particularly in this case, is needed to observe how informative a data visualization in delivering beneficial results to the users in making a decision [3]. The interface display in the form of data visualization becomes one of the important aspects of the success of a data analysis product.

One of the ways to make an evaluation is by using usability testing. The usability assessment still becomes an important step to be done as it is tightly related to the users' perception. Usability testing involves a set of planning, thinking, developing, and implementing process.

Usability can be defined as to what extent certain users can use a product to achieve the purpose determined by effectiveness, efficiency, and satisfaction in the context of predetermined utilization based upon [4]. Reference [2] defined usability based on five components:

1) Learnability: It deals with how simple the usability is for the users to accomplish the basic tasks when using the system.

2) Efficiency: It deals with how fast the users can accomplish the task once they learn the system.

3) Memorability: It deals with how simple the users can be skilful in using the system after they do not use it for a long time.

4) Errors: It deals with how many mistakes are made by the users and how simple they can handle them.

5) Satisfaction: It deals with whether the users are comfortable using the system and satisfied with its use. 


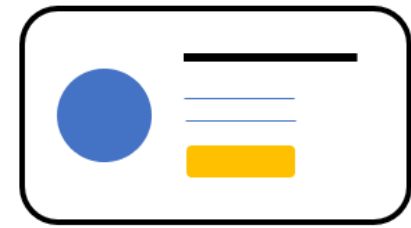

Step 1:

Click register button in

registration section

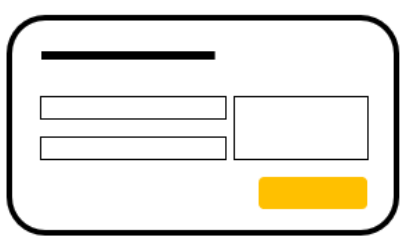

Step 2:

Filling user information

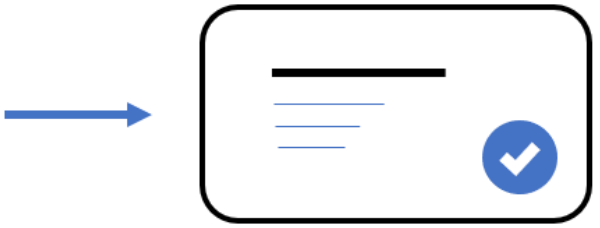

Step 3:

User receive feedback message

Fig. 1 The one-way flow of user registration.

Usability testing is a technique used to evaluate a product by doing a test on the user. This technique will give direct input about using the system to the users [5].

One of the ways to do usability testing is by directly asking the prospective users. Through the content of the questionnaires and the interview, the users' habit in using the system can be learned; it generates the input on several matters that can improve the system used for the users [5].

The scenario is one of the forms used in a usability test to understand how users experience the system. Commonly, it is an application in the form of a one-way scenario, for instance, buying a product, adding the data, registering as the site member, and other actions in which their flow can clearly be defined. This flow is called the user flow - a set of measures done by the users to reach a certain purpose. Fig. 1 shows the sample of the one-way user flow. At least, there are three fundamental requirements of a user flow.

1. A clear purpose the users must have.

2. One-way direction.

3. Representing an intact task.

The one-way linear flow will help to understand the users' habits in a system with a general aim found. However, when a system aims to give new knowledge through data analysis results, the one-way linear scenario model will be less optimal.

When the users access the data analysis dashboard, they have reached the final point of the flow and no longer need to explore page by page to reach a purpose. When being in the display of the data analysis dashboard, the user aims to explore, learn, interact, and make a conclusion on one page only. There is no specific task to be accomplished. How the users will use the system to reach their purpose will emerge several possibilities to diverge.

Therefore, a one-way linear scenario commonly used in the usability test will give a less optimal result in understanding the users' habits. This study proposed a modification in the components of a usability test scenario with the user story to evaluate the interface display of the data visualization dashboard.

\section{Problem with Common Usability Testing}

Some research on usability tests have been done. Reference [6] proposed an usability test implementation that was done automatically on some aspects and usability guidelines. Usability test automatically was done using the software with a pattern previously determined. The usability test pattern used in this study was made based on the Domain-Specific Language (DSL) that was possible to result in the testing model [7]. The testing model would be executed using the software to observe how a task's execution was conducted. For instance, the number of steps needed to access page A was three steps, but when the usability test was done automatically, it was found that to access page A, it needed four steps. From that result, it could be found that there was still a possibility that the users would do the things that are not suitable with the right pattern scenario (three steps).

Research shows the implementation of usability tests without the respondents' existence [6]. However, the usability test scenario based on the DSL becomes limited and unable to represent several scenarios that the users might do. In addition, the use of DSL patterns makes it possible to make mistakes in making the testing scenario excluded as the scenario of the usability test.

Ten usability heuristics as the parameter of assessment in conducting usability testing were proposed [8]. Reference [5] underlay the ten heuristics approach. The heuristic approach was made as the usability test assessment scenario used had the practical rules. Usability test with the heuristic assessment was done in four online sites of the university. This study concludes that the use of the heuristic parameter of usability test can improve the understanding of users' habits when using the application.

Reference [9] conducted the usability test on the online sites of the government portal. This study evaluated the use of the Ministry of Education of Malaysia's online site to measure the level of usability of the online portal site and proposed the improvement needed to be done based on the results of the evaluation. The usability test in this study was focused on seven attributes adopted from ISO/IEC 25010 and Website Analysis and Measurement Inventory (WAMMI), i.e., effectiveness, efficiency, learnability, controllability, attractiveness, helpfulness, and satisfaction. Two usability test phases were done, including a pre-usability test to evaluate the existing online site and a post-usability test done on the online site that has been improved based upon the results of the pre-usability test.

Commonly, research on usability test is conducted to observe the users' habits are towards certain application, for instance, on the online site of a university [8] with some pages containing the information and the online site of government information portal [9]. The user flow on this online site was 


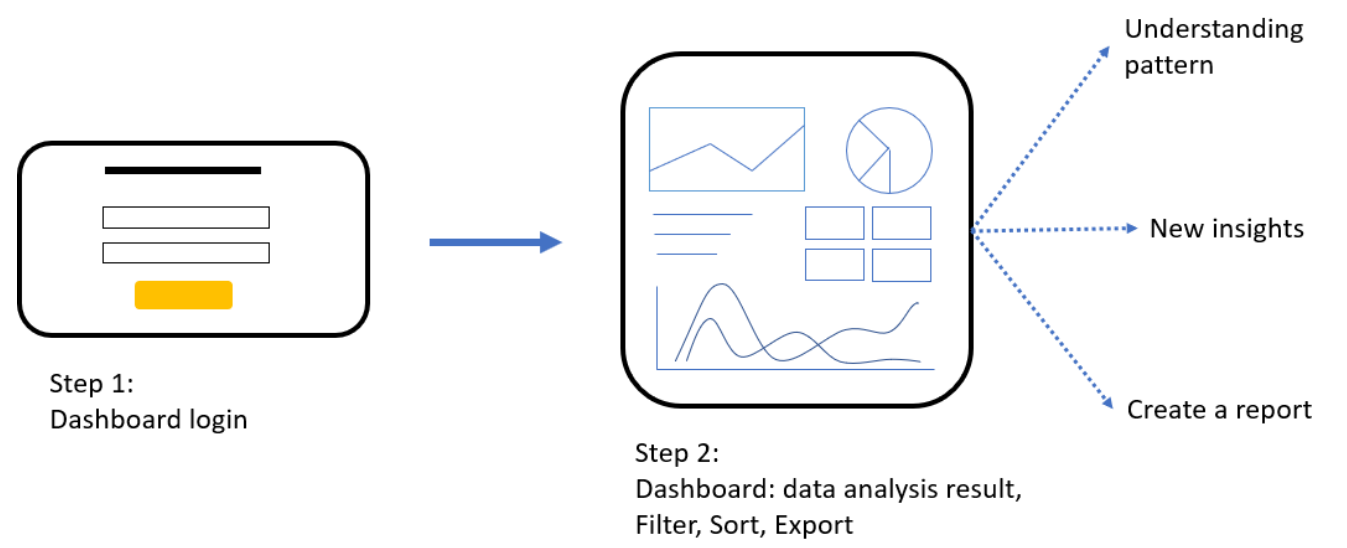

Fig. 2 Branching user flow.

TABLE I

THE COMPARISON BETWEEN COMMON DESIGN AND PROPOSED DESIGN

\begin{tabular}{|c|c|c|c|}
\hline \multicolumn{2}{|c|}{$\begin{array}{l}\text { The Common Design for } \\
\text { Usability Testing Consists of } \\
\text { the Following Components }\end{array}$} & \multicolumn{2}{|c|}{$\begin{array}{c}\text { Proposed Modified } \\
\text { Usability Testing Design for } \\
\text { Dashboard Data Analytics }\end{array}$} \\
\hline 1. & Item to validate & 1. & Item to validate \\
\hline 2. & Objective & 2. & Objective \\
\hline 3. & Method & 3. & Method \\
\hline 4. & User criteria & 4. & User criteria \\
\hline 5. & Metrics to measure & 5. & Metrics to measure \\
\hline 6. & Device & 6. & Device \\
\hline 7. & Research questions & 7 & Research questions \\
\hline 8. & Habit related questions & 8. & User stories \\
\hline 9. & Feature-based scenario & 9. & Story-based scenario \\
\hline 10. & Steps & 10. & Steps \\
\hline 11. & Findings & 11. & Findings \\
\hline
\end{tabular}

linear with one direction. The implementation of usability testing in this system with the branching users' flow, such as the data analysis system, was still limited in number. Fig. 2 illustrates the branching users' flow.

With the branching user flow, the commonly used usability testing scenario is no longer suitable with the characteristics of the data analysis system. Hence, this study proposes modifying the scenario on usability testing by using the user story to understand users' habits in using the data analysis system. The comparison between conventional and the proposed design of usability test is shown in Table I.

When a user accesses the data analysis dashboard, the user will see various information displayed in various forms. In those circumstances, the user's aim to analyze data will be wide and open to many possibilities. Such that there is no specific task to be done.

Since there is no specific task to be done, this modified usability test will help the user experience researcher define tasks aligned with the purpose of the data analytics system itself. When the tasks are defined properly, it will help the researcher to have a better understanding of the user experience and deeper understanding of the usability problem when using a data analysis system.

User story is used as the base to make the questionnaires related to the use of the system. These questionnaires will make the perception and experience of the users when using the system. Once the user's experience had been made, this study will evaluate and measure the user's experience using the technique of System Usability Scale (SUS). With that technique, it is expected that it can facilitate the users in using the data analysis dashboard system and then can provide the assessment in the system usability more accurately.

\section{ModifiEd UsABILITY TeSTING}

This study developed the data analysis system dashboard prototype displaying the data processing integrated with COVID-19 in Gorontalo Province. Data analysis was adjusted with the recent case of people health, including the information in terms of the update and the case's spread. In addition, there were the public sentiments based on the keyword "covid19," in which the system would take the data from a number of online sources: social media, online media, and some sites providing the open data. This prototype can be accessed online through the site browser at http://mediainsight.space.

The purposive random sampling approach was used for determining the participants. The participants in this study were active university students at the Department of Public Health of the State University of Gorontalo. This selection was based upon the system content related to the management of COVID19 in the local area, particularly in Gorontalo Province. In this case, the researcher considered that the students of Public Health were the domain master in line with the theme proposed in the integrated dashboard system.

One of the purposes of the usability test in this study was to detect the problems in using the interface of a dashboard system consisting of many graphs. Thus, the researcher determined the number of participants through the following three stages [10].

1. Observing the problems that might affect $10 \%$ of the users. This number was obtained with an assumption that the users have had the expertise in operating computer and had the knowledge background about the theme proposed in the dashboard system.

2. Estimating the percentage of problems that might emerge. Though the users have had the expertise in operating the computer, the system in the form of a data analysis dashboard was still found rare in use. The researcher estimated that approximately $85 \%$ of the problem could emerge. 
3. Based on the two values above, the researcher used the formula of binomial probability to determine the size of the sample $\log$ (1-problem that might emerge)/log (1-the affected users).

From the three phases above, the result of the calculation was 18.87. To rounded off the value and made it even, this study then made the value to 20 participants.

The implementation of usability test of the data analysis system was implemented in three phases. Adopted from [11], the phases of usability test included: a) making the test concept; b) making user story; c) validating the user story-based usability testing; and d) calculating the score of the usability test.

For instance, each of the phases was presented in detail on the feature of the date range filter to display the different data.

\section{A. Making the Test Concept}

To help in understanding the test concept to be done, the researcher attempted to answer the $5 \mathrm{~W} 1 \mathrm{H}$ questions:

1) What Features will be Tested?: One of the features to be tested was data filter of the COVID-19 case on March 2- March $25,2020$.

2) Why is the Test Needed to be Done?: Learning the insight obtained from the filter feature and understanding the habits of the users when doing the filter.

3) How to Do the Test?: The participants would get instructions from the facilitator to do the test. The questions from the facilitators can be proposed after the participants have done the test.

4) Who are the Targeted Participants?: As the data analysis system is expected to be widely used by the users, the targeted participants were the students of Public Health, understanding the domain of the data analyzed by the system.

5) How to Measure the Achievement?: The participants were able to decide from the data displayed and answer the number of COVID-19 cases in the range of time determined.

6) What will be Observed from this Test?: It dealt with whether the data displayed were able to give a new insight or a similar conclusion. In addition, it was asking if the users could do the data filter easily.

\section{B. Making Scenario - User Story}

Based on [12], there are two types of scenarios: exploratory tasks and specific tasks. This study used the scenario in the form of the exploratory task: the scenario was addressed to learn how the users could find or explore the information.

Such a scenario had no right answer, but it was oriented to the purpose. Exploratory tasks brought an impact on the type of branching user flow. For example, the data filter of COVID-19 cases in the range of March 2 to March 15, 2020, in the similar graph.

a. Policy Makers

(1) Purpose:

To design the policies for the next two weeks.

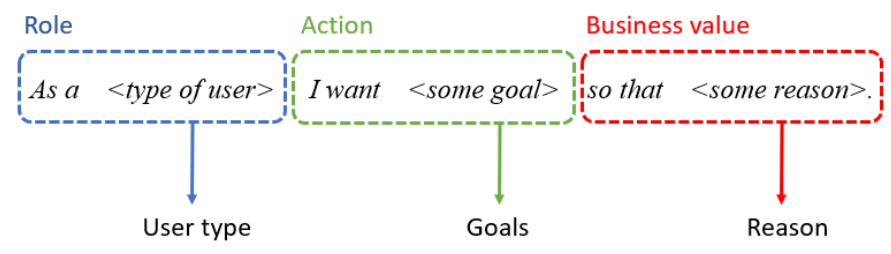

Fig. 3 A general format of a user story.

(2) User story:

With the exponentially more increasing cumulative cases day by day, what measure must be taken to minimize the transmission of the COVID-19 virus for the next two weeks?

b. Health Workers

(1) Purpose:

To prepare health workers and medical infrastructure.

(2) User story:

The exponentially increasing number of cumulative COVID-19 cases must be supported managed with the medical workers' preparedness and medical infrastructures for the next times.

c. Public

(1) Purpose:

To observe how to prepare themselves.

(2) User story:

The cumulative increase of cases from 34 cases to 69 positive cases indicated that the virus of COVID-19 could spread easily. People must initiate to keep themselves away from the transmission of COVID-19.

User story refers to the short sentence containing the requirements and functions required by the users. Fig. 3 shows the format commonly used in the user story [13]. Each part of the user story above is defined as follows:

1. <type of user> represents the users of the system. In this case, the type of user can be in the form of a policymaker, medical worker, or community,

2. <some goal> represents the purpose of the users in using the system, and

3. <some reason> represents the reason for the users to reach the purpose.

Before making the user story, this research asked the health experts how an integrated data system could help manage the pandemic. At this phase, the researcher prepared the mockup containing the proposal features and explained the system's flow. These features were determined based on the initial user story and implemented using the design thinking method by taking several references from the existing system and by modifying based upon the temporary needs.

Having obtained the experts' input, the researcher improved the display and features to meet the needs of an integrated data system to support the management of the pandemic. User story was then made by following the scenario that might occur when this system was used by the health workers, policymakers, or community.

In making the user story, the researcher attempted to understand the structure of forming the user story utilizing the 


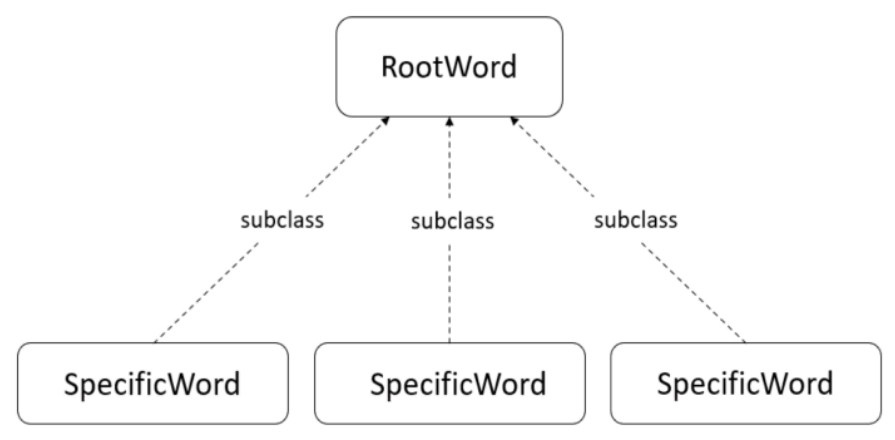

Fig. 4 The illustration of the ontology in one set of words.

ontology - a formal model assisting to describe the concept in a certain domain [14]. Ontology plays a role as the base of knowledge about each word's terms and relation [15].

In each set of word role, action, and the business value, it would be added with the class and hierarchy as the concept of basic knowledge in an ontology. The hierarchy of words is illustrated with the tree model with the root word and derivative words called specific words. Fig. 4 displays the illustration of the ontology of a set of words.

Based on the general format of a user story, the ontology hierarchy model was formed as Role - Action - Business value. Fig. 5 illustrates the ontology hierarchy.

\section{Validating the User Story-Based Usability Testing}

An experiment was conducted to validate the proposed method. The experiment was done by developing the system's prototype version and involving participants to answer questions using the system. These questions were designed based on the user story as a "job to be done" for a user. The answers to the questions were tools to measure user ability in turning information into insights. After they answered, participants were also asked to assess the acceptability of the system using SUS. This research conducted a controlled experiment that started from user recruitment until the end of the experiment activities.

Before the experiment was held, the researcher asked participants to make a statement that they would complete the questionnaire and asked their permission to use their behavior data during the experiment.

The main step of the experiment was usability testing. The researcher split the group of participants into two groups and gave a different experiment schedule for each group. Each group contains ten participants. Participants gathered in a private house and obeyed the health protocols during the pandemic; the researcher made sure that each participant does not have a high body temperature and implemented strict protocols such as washing hands before entering the experiment area and maintaining the distances and wear a mask.

Logistics was prepared to support the experiment. Each participant should bring their laptop, and at least there was one browser installed. The researcher provided them with internet access, which could be accessed for each participant. Moreover, each participant would receive a questionnaire document generated based on the user story. It included the scenarios that might be happened for each role as a health worker. The usability test was conducted in the following steps.

1. The researcher did a short presentation about the experiment and the data analysis system they would use during the experiment. It includes explaining the aim of the system, the research domain, and what to measure.

2. Participants had15 minutes to overview the system.

3. Answering the questionnaires inside the document. These questionnaires were generated based on the user story. By answering these questions, the user would build the user experience. Participants could answer these questions by using the system. The researcher assessed the document based on the correct answer. If all questions were answered correctly, they would receive a score of 100 . The score's calculation was by subtracting the total questionnaires with wrong answers. Divide the subtracted value with the total questionnaires and multiply by 100 to receive the final score. For example, with two wrong answer: $30-2=28$; final score: $(28 / 30) \times 100=93.4$.

4. After the user experience was built, the researcher asked the participants to fulfill the system usability scale. In this phase, participants were supposed to develop their experience, which would affect their perception on the usability scale.

\section{Calculating the Scores of Usability Test}

After the usability test was done, the participants would be asked to fill the questionnaires about the System Usability Scale (SUS). The questionnaires contained ten questions based upon the general template of SUS. Each of the questions was measured based on the Likert Scale, started from very disagree scored 1 to very agree scored 5 .

Attributes to be measured used the SUS questionnaire consisting of effectiveness, efficiency, learnability, controllability, attractiveness, helpfulness, and satisfaction. The formula for calculating SUS is presented below.

$$
\bar{x}=\frac{\sum x}{n}
$$

where $\bar{x}$ refers to the mean score, $\sum x$ refers to the weight of scores of SUS, and $n$ : refers to the respondents. Given the usability score, it was expected to be the parameter of how good the data analysis system was in delivering the information or insight to the users.

\section{EXPERIMENTAL RESULTS AND DISCUSSION}

The scenario modification in the usability test was done by changing the one-way scenario into a user story. With this change, the researcher wanted to embed the idea for the participants that users had a share in determining a data analysis system's success. The exploration of data from analysis results could be utilized in various ways to increase system usability.

One of the user story formulation techniques in this study was to map a user story's ontology. It started by understanding each basic word component and reducing it to be more specific words. After finding a suitable word composition, a set of words were combined into a sentence indicating a user story. 


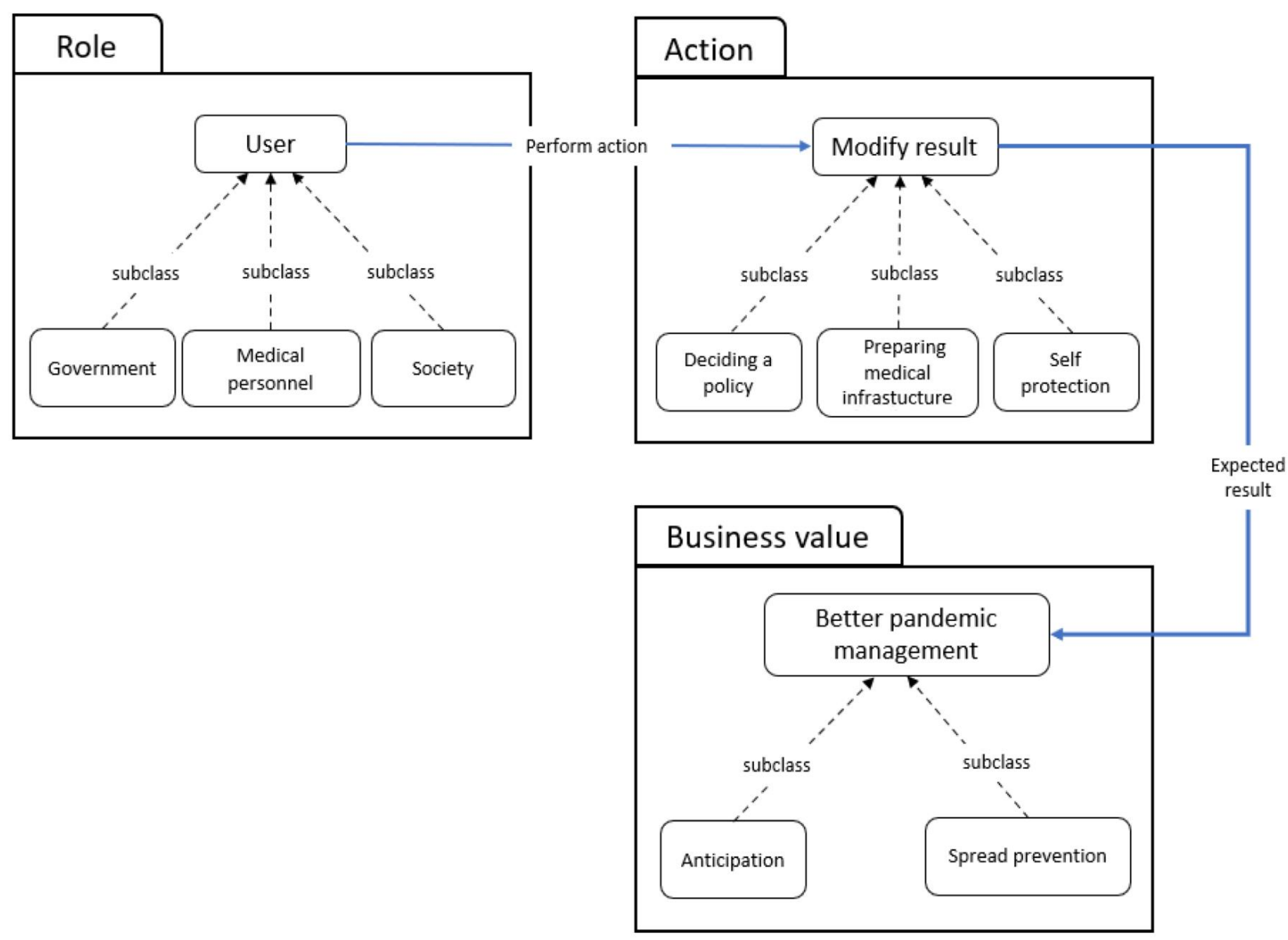

Fig. 5 The sample of the ontology hierarchy of the user story for COVID-19 analysis data.

This technique was expected to facilitate the formulation of user stories.

The user story components at the usability test stage played an important role in determining the delivery of information from data analysis through visualization. The usability test was done in such a way to enable the participants to understand the potential probably raised from using a data analysis system. Then, to validate the idea, a usability test score was calculated by considering several predetermined aspects.

User story resulted as follows.

1. User story 1:

"The exponential increase in the number of cumulative cases of COVID-19 must be accompanied by the readiness of medical workers and medical infrastructure. As a regional health worker, I want to know the condition of COVID-19 in the city where I am living."

a. Role: "As a local health worker ..."

b. Action: "... I want to know the condition of COVID19 in the city where I am living in."

c. Business value: "Preparing medical personnel and infrastructure."

The number of resulted questionnaires: 17 .

2. User story 2:

"COVID-19 is a contagious disease easily be spread by a person infected with the virus. It causes the rate of positive cases to grow very fast. Given the nature of transmission, information about mass movement and tracking of positive cases is deemed essential to anticipate and control transmission."

a. Role: "Regional BNPB, Police, Health Service"

b. Action: “... Case tracking and mass movement information."

c. Business value: "anticipating and controlling transmission"

The number of questionnaires resulted: 6 .

3. User story 3:

"As a public health worker, I want to know public sentiments about the COVID-19 pandemic through social media to help the government in making public policy."

a. Role: "public health worker"

b. Action: "... recognizing public sentiment towards the COVID-19 pandemic"

c. Business value: "assisting the government in making public policy"

The number of questionnaires resulted: 7 .

Every action generated from three user stories was assumed to be done by participants by answering all the 30 questions. Information on public sentiment is presented through a time series graph display where the $\mathrm{x}$-axis refers to the date, and the $\mathrm{y}$-axis refers to the number of sentiments. 


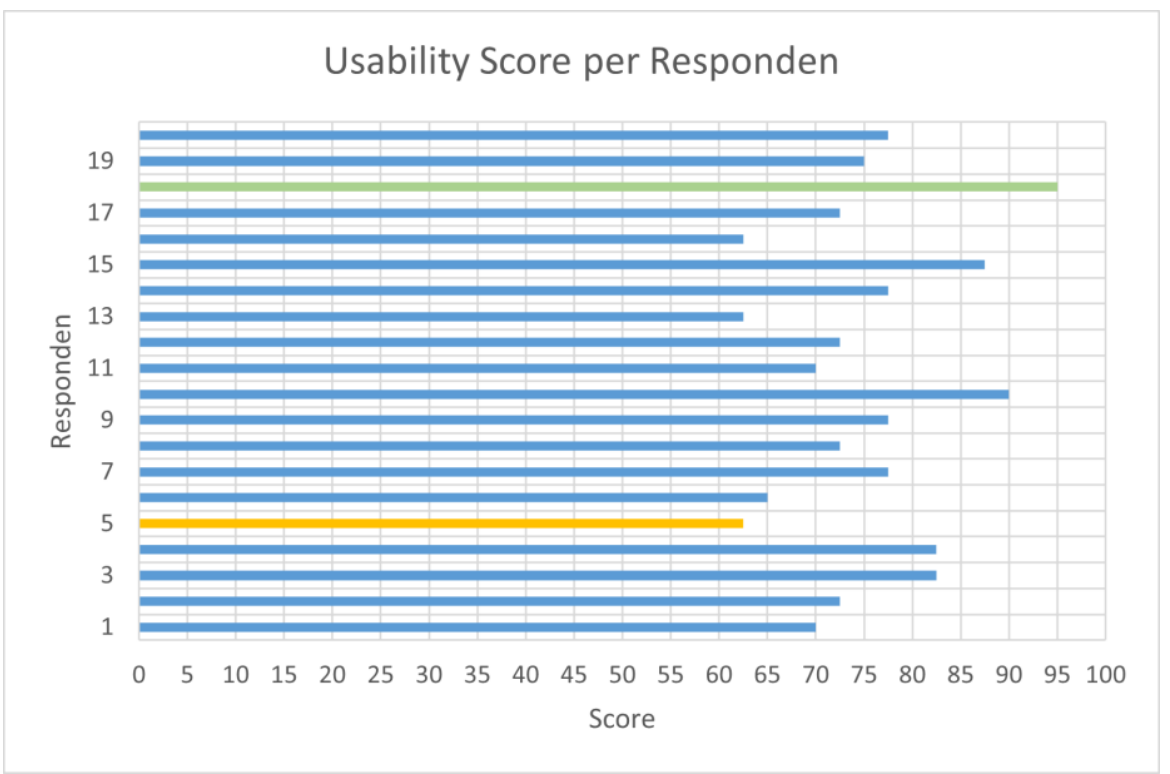

Fig. 6 Score of System Usability Scale (SUS) per person.

\begin{tabular}{|c|c|c|c|}
\hline & Not Acceptable & Marginal & Acceptable \\
\hline & Worst Imaginable & Ok & Good Excellent Best Imaginable \\
\hline
\end{tabular}

Grade
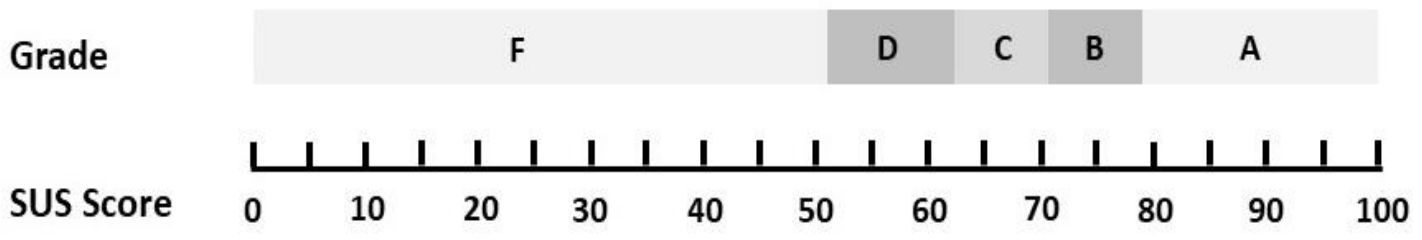

Fig. 7 Grades, adjectives, acceptability, categories associated with raw SUS scores.

In the graphic display, there is a feature to sort the information. This feature is provided to make it simple for the users to gain new knowledge about how public sentiment is during the pandemic. In user story 3 , users were asked to know what date there was a change in people's emotions from happy to sad. To facilitate getting this knowledge, users could sort out the sentiments to be compared. However, most users did not provide the exact answers about when changes in public sentiment occurred. This was because users did not use the available data sorting feature, so they did not get the expected knowledge.

In user story 1 , there was information about the province's emergency status, which users should easily know. However, many users made mistakes when acquiring this knowledge. When the users reached the end of the page, the users were no longer compelled to seek information at the beginning. Moreover, in the middle of the page, there was a component displaying the district's status. This component provided a misinterpretation of the status of the provincial emergency response. The high number of graphic components on a page could make it difficult for users to identify specific information.
From the researchers' observations, it is important to explain each available feature to the users in more detail. In addition, researchers also observed the time needed by users to understand the usability of the features available in more detail.

The data sorting feature in the interactive time series graphics was not utilized by most users, so it caused many errors in gaining the knowledge requested in the user story. It was confirmed by the observations of the two points of the SUS questionnaire. This result indicates that users need help and time to get used to using the system.

After calculating the SUS score of 20 respondents, the highest SUS score was found at 95, and the lowest score was at 63. Meanwhile, the mean score of SUS obtained was 75.25. The SUS score can be interpreted in the form of a system usability classification. The score of SUS is shown in Fig. 6.

As seen in Fig. 7, the score obtained is categorized as a good product with a B grade scale. However, since the average SUS score in the study is above the mean score of SUS of 68, it can be considered acceptable [16].

In order to ascertain that users could get knowledge of the given scenario, the researcher assessed each score obtained by the participants based on the correct answer. Of the three user 
stories with a total of 30 questionnaire questions, the average score obtained from 20 participants was 91.8 . With this score, it can be concluded that the system interface is able to convey information well; thus, the users can obtain new knowledge about the of COVID-19 management in Gorontalo.

\section{CONCLUSION}

The branching user flow in using the data analysis system became the background to modify the components of the scenario in the usability test. Steps of a usability test with scenario change of the linear flow into the user story have been presented in detail. The use of ontology-based on the basic format of the user story is expected to facilitate in formulating the user story.

Given the user story, the orientation of the users would be built based upon the expected purpose. Thus, each user could more understand each step that must be done to reach a purpose. Having conducted using the user story scenario, the researcher, by using the specific feature, was able to identify if it was still difficult for the users. Of the total 30 questions in the questionnaires, 20 participants answered with an average score of 91.8. It indicates that the users are able to find insight as one of the research purposes. Then, the mean score of SUS was obtained at 75.25 with good and acceptable categories.

In addition to SUS, the user experience measurement can be measured using the Net Promoter Score (NPS). Principally, NPS measures whether the users will recommend the system to other users. There is a strong correlation between SUS and NPS. The SUS score at least must approach the number 81 to reach the recommendation or high NPS score. At the same time, this research has not achieved the recommended score yet. The next research is expected to increase the SUS score to be categorized equally to the category of NPS promoter in which this system brings a good impact and can be recommended by the users to be used by others.

\section{REFERENCES}

[1] S.K. Sahu, M.M. Jacintha, and A.P. Singh, "Comparative Study of Tools for Big Data Analytics: An Analytical Study," 2017 Int. Conf. on Comp.,
Comm. and Autom. (ICCCA), 2017, pp. 37-41.

[2] J. Nielsen, Usability Engineering, San Fransisco, USA: Morgan Kaufmann, 1993.

[3] Y. Qi, G. Shi, X. Yu, and Y. Li, "Visualization in Media Big Data Analysis," Proc. 2015 IEEE/ACIS 14th Int. Conf. Comput. Inf. Sci. (ICIS 2015), 2015, pp. 571-574.

[4] "Systems and Software Engineering - Systems and Software Quality Requirements and Evaluation (SQuaRE) — System and Software Quality Models," British Standards Institution, 2013.

[5] J. Nielsen, "Usability Assessment Methods beyond Testing," in Usability Engineering, San Fransisco, USA: Morgan Kaufmann, 1993, pp. 207-226.

[6] F. Dias and A.C.R. Paiva, "Pattern-Based Usability Testing," 2017 IEEE Int. Conf. on Software Testing, Verification and Validation Workshops (ICSTW), 2017, pp. 366-371.

[7] R.M.L.M. Moreira and A.C.R. Paiva, "A GUI Modeling DSL for PatternBased GUI Testing PARADIGM," 2014 9th Int. Conf. on Eval. of Novel Appr. to Software Eng. (ENASE), 2014, pp. 1-10.

[8] A. Lodhi, "Usability Heuristics as an Assessment Parameter: For Performing Usability Testing," 2010 2nd Int. Conf. on Software Technol. and Eng., 2010, pp. 256-259.

[9] N. Binti N. Rozali and M.Y. Binti Said, "Usability Testing on Government Agencies Web Portal: A Study on Ministry of Education Malaysia (MOE) Web Portal," 2015 9th Malaysian Software Eng. Conf. (MySEC), 2015, pp. 37-42.

[10] J. Sauro (2011) "How to Find the Right Sample Size for a Usability Test," [Online], https://measuringu.com/sample-size-problems/, access date: 24-Sep-2020.

[11] J. Nasaputra (2020) "Usability Test \#2: Merencanakan UT dalam 3 Tahap,” [Online], https://medium.com/belajar-desain/usability-test-2 merencanakan-ut-dalam-3-tahap-d419c2cffd6b, access date: 25-Sep2020.

[12] H. Loranger (2016) "Checklist for Planning Usability Studies," [Online], https://www.nngroup.com/articles/usability-test-checklist/, access date: 25-Sep-2020.

[13] M. Cohn, User Stories Applied: For Agile Software Development, 1st ed Boston, USA: Addison-Wesley Professional, 2004.

[14] D. Leffingwell, Agile Software Requirements: Lean Requirements Practices for Teams, Programs, and the Enterprise, Boston, USA: Addison-Wesley Professional, 2010.

[15] P. Abrahamsson, O. Salo, J. Ronkainen, and J. Warsta, Agile Software Development Methods: Review and Analysis, Vuorimiehentie, Finland: Julkaisija - Utgivare - Publisher, 2002.

[16] J. Brooke, "SUS: A Retrospective," J. Usability Studies, Vol. 8, No. 2, pp 29-40, Feb. 2013. 